\title{
Assessing risk is the business of prion disease research
}

The December 23, 2003, announcement of the first US case of mad cow disease raised the public's sense of alarm about beef safety, deflated beef stock options prices, and opened a new discourse among US policy makers and scientists about the aims of research into this perplexing disease.

Mad cow disease, or bovine spongiform encephalopathy (BSE), is one of several transmissible spongiform encephalopathies (TSEs). These diseases are caused by an altered structural form of the normal cellular prion protein. Transmission of this aberrant protein form can occur through various means, including the ingestion of

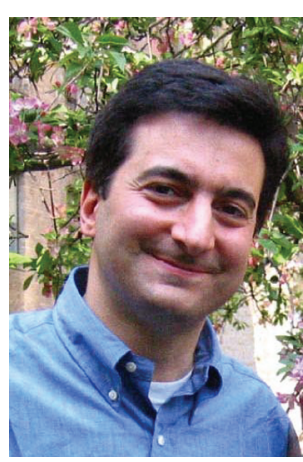

Robert Klitzman asks how society deals with risk. contaminated food.

This last possibility, and the knowledge that BSE had entered human food sources in the UK and elsewhere in Europe and had led in the 1990s to a variant form of Creutzfeldt-Jakob disease (vCJD) in humans, has raised public awareness and fears of the potential widespread risk of this disease in humans.

Robert Klitzman of Columbia University advises that the relative risk to the US population remains unknown but that understanding that risk is essential for making research policy.

Klitzman told the JCI, "It would not surprise me if there were other cattle in the US from Canada that [are] infected." From previous studies he notes that, "epidemiologically, we know there is a bell-shaped curve for the incubation [of this disease].” Thus, this single case of BSE in the US may be only the beginning. Klitzman hopes, however, that the US will be lucky and this will be the only case.

The real question for health officials is whether BSE in the US cattle population will translate into human disease. Klitzman's work on kuru in New the need for such studies.
Guinea makes clear the difficulty of making such a prediction. The Fore people had held ritual cannibalistic feasts in which deceased relatives, including their brains and viscera, were eaten. Klitzman found that there were individuals who had attended many such feasts but never got sick, whereas others who had been to a single feast had eventually fallen ill, up to 45 years later. "This tells us two things," Klitzman says, "that oral uptake [of the disease] is not that high, but that it can occur."

The total number of reported cases of $\mathrm{VCJD}$ in the UK between 1996 and 2002 was 129 , and there were thousands of cattle with BSE. With only one

Currently, testing for BSE can only be done postmortem and focuses on cattle that were unable to walk or that have died of unknown causes. All such animals are inspected for BSE pathology in one of the network labs and, if BSE is suspected, by the USDA.

Williams thinks the network is effective, stating, "I really do believe that if there were a lot of cases, they would be found." Testing every animal that goes to slaughter, as some have suggested, would be extremely costly. She is not convinced the current risks warrant such action.

Klitzman agrees and indicated that what we are prepared to do for public health in relation to BSE all depends on the amount of risk we are prepared to take and the cost we are prepared to bear.

"Do we want the risk to be zero?" Klitzman asks, "Or do we want some risk? Currently the public outcry is no [risk]. There is a price tag that has not yet been attached to this. If it is $\mathrm{X}$ percent more expensive to buy beef, the public may, at a certain point, say it is not worth it."

Here Klitzman raises an additional area of research that he feels is essential. There is a clear need, he told the JCI, "to do social science research on how we [as a society and as individuals] respond to risk." Coming to such an understanding could cer-

She told the JCI that we need to assess how the agent works both in individual animals and in populations, and that it would be difficult to make policy or create a targeted research program without such information.

Williams' lab is one of a large network involved in the surveillance of BSE in the United States since the beginning of the 1990s, a network that Williams feels works very well in monitoring and detecting BSE in the US. tainly aid in handling the current concerns about BSE in the US beef supply, but also in determining funding and directives for research for the variety of disorders where the probable and perceived risks are at odds.

\section{Laurie Goodman, New York}

\footnotetext{
1. 2004. Advancing prion science: guidance for the Nation al Prion Research Program. R. Erdtmann and L. Sivitz, editors. National Academies Press. Wash ington, DC, USA. In press.
} 